\title{
Seletividade inicial de herbicidas aplicados em pós-emergência da crotalária ${ }^{1}$
}

\section{Initial selectivity of herbicides applied in post-emergence of showy crotalaria}

\author{
Roque de Carvalho Dias ${ }^{2}$; Kassio Ferreira Mendes ${ }^{3}$; Clebson Gomes Gonçalves ${ }^{4}$; Christiane \\ Augusta Diniz Melo ${ }^{5}$; Matheus Ferreira França Teixeira ${ }^{6}$; Daniel Valadão Silva ${ }^{7}$; Marcelo \\ Rodrigues Reis ${ }^{8}$
}

Resumo - Dentre as práticas que visam conferir sustentabilidade do solo agrícola, o emprego de adubos verdes e/ou plantas de cobertura como a crotalária tem sido preconizado. O manejo de plantas daninhas é uma das práticas para o sucesso da crotalária. Entretanto, as informações sobre a seletividade de herbicidas são escassas, sendo estas de suma importância. Assim, objetivou-se avaliar a seletividade de herbicidas aplicados em pós-emergência da crotalária (Crotalaria spectabilis). Foram realizados dois experimentos em casa de vegetação no delineamento inteiramente casualizado, com quatro repetições. No primeiro foram testados os seguintes herbicidas ( $\mathrm{g} \mathrm{ha}^{-1}$ i.a.): atrazine (500 e 1000), bentazon (720), carfentrazone-ethyl $(3,75)$, clomazone (400), diclosulam (20), diuron (800), ethoxysulfuron (18), flumioxazin (10), mesotrione (60), oxadiazon (500), tembotrione (40), atrazine + flumioxazin + carfentrazone-ethyl (300 + 5+ 2), e uma testemunha sem aplicação. No segundo aplicou-se: bentazon (400), clomazone (400), diclosulam (10 e 15), diuron (400), ethoxysulfuron (15), bentazon + diclosulam $(200+7,5)$, diclosulam + diuron $(7,5+200)$, bentazon + diuron $(200+200)$, e uma testemunha sem aplicação. Foram realizadas avaliações de fitotoxicidade aos 7, 14, 21 e 28 dias após a aplicação (DAA) e de matéria seca da parte aérea (MSPA) aos 28 DAA. Em ambos os experimentos os herbicidas bentazon, clomazone, diclosulam e ethoxysulfuron causaram baixa fitotoxicidade à cultura e baixa redução na MSPA. Os herbicidas atrazine, mesotrione, oxadiazon e atrazine + flumioxazin + carfentrazone-ethyl proporcionaram altos níveis de intoxicação e alta redução na MSPA. Os herbicidas bentazon, clomazone, diclosulam e ethoxysulfuron foram seletivos para aplicação em pós-emergência da cultura.

\footnotetext{
${ }^{1}$ Recebido para publicação em 12/01/2017 e aceito em 22/02/2017.

${ }^{2}$ Universidade Estadual Paulista (UNESP), Faculdade de Ciências Agronômicas, Rua: José Barbosa de Barros, $\mathrm{n}^{\circ}$ 1780, CEP: 18610-307. Botucatu, São Paulo, Brasil. E-mail: <roquediasagro@gmail.com>.

${ }^{3}$ Laboratório de Ecotoxicologia, Centro de Energia Nuclear na Agricultura (CENA), Universidade de São Paulo USP, Avenida: Centenário, ${ }^{\circ}$ 303, São Dimas, CEP: 13416-000. Piracicaba, São Paulo, Brasil. E-mail: <kassio_mendes_06@hotmail.com>.

${ }^{4}$ Universidade Estadual Paulista (UNESP), Faculdade de Ciências Agrárias e Veterinárias, Via de Acesso Prof. Paulo Donato Castelane, s/n - Vila Industrial, CEP: 14884-900. Jaboticabal, São Paulo, Brasil. E-mail: <goncalvescg.agro@hotmail.com>.

${ }^{5}$ Universidade Federal do Triângulo Mineiro (UFTM), Campus Iturama, Avenida: Rio Paranaíba, no 1241 , Centro, CEP: 38280-000. Iturama, Minas Gerais, Brasil. E-mail: <chrisadinizmelo@yahoo.com.br>.

${ }^{6}$ Universidade Federal de Viçosa (UFV), Campus Viçosa, Avenida: Peter Henry Rolfs, s/n - Campus Universitário, CEP: 36570-900. Viçosa, Minas Gerais, Brasil. E-mail: <teixeiramff@gmail.com>.

${ }^{7}$ Universidade Federal Rural do Semi-Árido - (UFERSA), Avenida: Francisco Mota - Pres. Costa e Silva, CEP: 59625300. Mossoró, Rio Grande do Norte, Brasil. E-mail: <danielvaladaos@yahoo.com.br>.

${ }^{8}$ Universidade Federal de Viçosa (UFV), Campus Rio Paranaíba, Rodovia MG-230 - Km 7, CEP: 38810-000. Rio Paranaíba, Minas Gerais, Brasil. E-mail: <marceloreis@ufv.br>.
} 
Palavras-chaves: adubação verde; Crotalaria spectabilis; intoxicação por herbicidas; manejo de plantas daninhas

Abstract - Among the practices that aim to confer sustainability of the agricultural soil, the use of green manure and/or cover crops such as crotalaria are advocated. The management of weeds is one of the practices for the success of crotalaria. However, the information about the selectivity of herbicide are scarce and these are of paramount importance. The objective of this study was to evaluate the selectivity of different herbicides applied in post-emergence of showy crotalaria (Crotalaria spectabilis). Two experiments were conducted in a greenhouse in a randomized completely randomized design with four replications. In the first one the following herbicides were tested. The first experiment involved the following herbicides (g. i.a.ha ${ }^{-1}$ ): atrazine (500 and 1000), bentazon (720), carfentrazone-ethyl (3.75), clomazone (400), diclosulam (20), diuron (800), ethoxysulfuron (18), flumioxazin (10), mesotrione (60), oxadiazon (500), tembotrione (40), atrazine + flumioxazin + carfentrazone-ethyl $(300+5+2)$, and a control without application. In the second were used, bentazon (400), clomazone (400), diclosulam (10 and 15), diuron (400), ethoxysulfuron (15), bentazon + diclosulam $(200+7.5)$, diclosulam + diuron $(7.5+200)$, bentazon + diuron $(200+200)$ and a control without application. Phytotoxicity was evaluated at 7, 14, 21 and 28 days after application (DAA) and aerial dry matter (ADM) at 28 DAA. In both experiments the herbicides bentazon, clomazone, diclosulam and ethoxysulfuron caused low injuries to the crop and low reduction in ADM. The herbicides atrazine, mesotrione, oxadiazon and atrazine + flumioxazin + carfentrazone-ethyl provided high levels of injuries and high reduction in ADM. The herbicides bentazon, clomazone, diclosulam and ethoxysulfuron were selectivity for postemergence application of the crop.

Keywords: green manure; Crotalaria spectabilis; intoxication by herbicide; weed management

A expansão da área ocupada pela agricultura tem levado muitas vezes a práticas de manejo do solo intensivas e não adaptadas às condições edafoclimáticas. Em consequência, a degradação física, química e biológica do solo tem aumentado, o que reflete em possíveis quedas de produtividade e gera um elevado custo econômico e ambiental (Erasmo et al., 2004).

Dessa forma, a adoção de práticas de manejo do solo torna-se essencial visando assegurar a sustentabilidade dos sistemas agrícolas, sendo importante a manutenção e o incremento de matéria orgânica no solo (Meschede et al., 2007). Assim, destaca-se a adubação verde como uma das práticas mais viáveis e eficientes para atender essa necessidade. Segundo Sagrillo et al. (2009), os adubos verdes apresentam boa produção de biomassa e sistema radicular profundo, o que auxilia na descompactação do solo e na economia com fertilizantes nitrogenados, especialmente com o plantio de leguminosas. Há também a melhoria da qualidade do solo, relacionado ao aumento do teor de matéria orgânica, à maior disponibilidade de nutrientes e à capacidade de troca de cátions efetiva do solo (Calegari et al., 1993).

Dentre as espécies de plantas utilizadas para fins de adubação verde destacam-se as leguminosas. De acordo com Silva e Menezes (2007) as leguminosas têm sido mais usadas como adubo verde devido à sua rusticidade, à elevada produção de matéria seca, ao sistema radicular profundo e à simbiose com bactérias fixadoras do $\mathrm{N}_{2}$ atmosférico.

A adoção da adubação verde também pode interferir no manejo integrado de plantas daninhas, suprimindo reinfestações, além de diminuir o banco de sementes. Neste sentido, o cultivo de crotalária pode provocar modificações na população de plantas daninhas, 
devido aos efeitos alelopáticos e competição por luz, água, oxigênio e nutrientes, acarretando na supressão de algumas delas (Adler e Chase, 2007, Monquero et al., 2009). A crotalária tem efeito supressor sobre as espécies de plantas daninhas, independente dos métodos de semeadura (Timossi et al., 2011).

Neste sentido, Crotalaria spectabilis Roth, da família Fabaceae destaca-se por possuir benefícios amplamente relatados na agricultura, como aporte de nutrientes no solo (Santos et al., 2010), produção de palha para o plantio direto (Gitti et al., 2012) e controle de fitonematoides, por produzir substâncias como a monocrotalina (Wang et al., 2002). Além disso, a crotalária também apresenta potencial para uso em sistemas de consórcio de culturas como o milho ou de outras espécies que podem ter a função de adubo verde, como o milheto, por exemplo. Entretanto, não existem herbicidas registrados para cultura da crotalária no mercado brasileiro de defensivos agrícolas (Oliveira Neto et al., 2011).

Diante dos benefícios da utilização da crotalária no sistema de rotação de culturas, torna-se fundamental a viabilização de práticas culturais para assegurar a sua produção, a exemplo do manejo de plantas daninhas. Entretanto, informações referentes à utilização de herbicidas seletivos a crotalária ainda são escassas, impossibilitando maior expansão desta cultura. Haja vista que em extensas áreas de cultivo da crotalária no Brasil, a não utilização do controle químico dificulta o manejo da comunidade infestante.

Com base nestas informações, o trabalho teve como objetivo avaliar a seletividade inicial de herbicidas aplicados em pós-emergência na cultura da crotalária (Crotalaria spectabilis).

Foram realizados dois experimentos em casa de vegetação em delineamento inteiramente casualizado, com quatro repetições constituídas de vaso plástico com capacidade para $8 \mathrm{dm}^{3}$ de solo. O solo utilizado para o preenchimento dos vasos foi proveniente de área que apresentou classificação de Latossolo Vermelho-Amarelo Distróférrico (EMBRAPA, 2013), de textura argilosa (Tabela 1).

Tabela 1. Atributos físico-químicos de Latossolo Vermelho distroférrico, textura argilosa, utilizada no experimento.

\begin{tabular}{|c|c|c|c|c|c|c|}
\hline \multicolumn{2}{|c|}{$\begin{array}{c}\mathrm{pH} \\
\left(\mathrm{H}_{2} \mathrm{O}\right)\end{array}$} & $\mathrm{Al}^{3+}$ & $\begin{array}{r}\mathrm{H}^{+}+\mathrm{Al}^{3+} \\
-\left(\mathrm{cmol}_{\mathrm{c}}\right. \\
\end{array}$ & $\mathrm{Ca}^{2+}+\mathrm{Mg}^{2+}$ & $\mathrm{Ca}^{2+}$ & $\begin{array}{c}\mathrm{K}^{+} \\
\left(\mathrm{mg} \mathrm{dm}^{-3}\right)\end{array}$ \\
\hline \multicolumn{2}{|c|}{6,00} & 0,00 & 3,50 & 4,60 & 3,60 & 100 \\
\hline $\begin{array}{c}\text { M.O. } \\
\left(\operatorname{dag~dm}^{-3}\right)\end{array}$ & $\begin{array}{c}\mathrm{P} \\
\left(\mathrm{mg} \mathrm{dm}^{-3}\right)\end{array}$ & $\begin{array}{l}\text { CTC }(\mathrm{pH}=7) \\
\left(\mathrm{cmol}_{\mathrm{c}} \mathrm{dm}^{-3}\right)\end{array}$ & (\% & Areia & $\begin{array}{l}\text { Silte } \\
\left(\text { dag kg }^{-1}\right)\end{array}$ & Argila \\
\hline 2,40 & 13,90 & 8,37 & 58,00 & 32,90 & 11,70 & 55,40 \\
\hline
\end{tabular}

Extratores: P e K - Mehlich 1; Ca, Mg, $\mathrm{Al}$ - KCl $1 \mathrm{~mol} \mathrm{~L}^{-1} ; \mathrm{H}+\mathrm{Al}$ - $\mathrm{Ca}(\mathrm{OAc})^{2}$ 0,5 mol L-1.

No Experimento 1 foram utilizados 13 tratamentos, além de uma testemunha sem aplicação (Tabela 2). Após selecionar os potenciais herbicidas seletivos para a crotalária foi conduzido o Experimento 2 para certificar a viabilidade do uso dos herbicidas e, ainda, considerar possíveis efeitos de doses e misturas. Neste trabalho foram utilizados: bentazon (400 $\left.\mathrm{g} \mathrm{ha}^{-1}\right)$; clomazone (400 $\left.\mathrm{g} \mathrm{ha}^{-1}\right)$; diclosulam (10 e $15 \mathrm{~g} \mathrm{ha}^{-1}$ ); diuron (400 $\mathrm{g} \mathrm{ha}^{-1}$ ); ethoxysulfuron $\left(15 \mathrm{~g} \mathrm{ha}^{-1}\right)$; bentazon + diclosulam $(200+7,5+$ $\left.\mathrm{g} \mathrm{ha}^{-1}\right)$; bentazon + diuron $\left(200+200 \mathrm{~g} \mathrm{ha}^{-1}\right)$; diclosulam + diuron $\left(7,5+200 \mathrm{~g} \mathrm{ha}^{-1}\right)$, além de uma testemunha sem aplicação.

A escolha dos herbicidas e doses foi baseada no trabalho de Braz et al. (2015) e na recomendação dos produtos registrados para a cultura do milho (Zea mays L.), visando o consórcio milheto/crotalária, além da eficiência no controle de plantas daninhas e do risco de causar intoxicação na cultura.

Foram semeadas em cada unidade experimental cinco sementes de crotalária na profundidade de $2-3 \mathrm{~cm}$ e aos sete dias após a 
emergência (DAE) foi realizado o desbaste mantendo-se apenas duas plantas por vaso. Durante a condução dos experimentos os vasos foram irrigados sempre que necessário visando manter a umidade próxima à capacidade de campo.

Tabela 2. Relação dos tratamentos e doses utilizadas no Experimento 1.

\begin{tabular}{lcc}
\multicolumn{1}{c}{ Tratamentos* $^{*}$} & Nome comercial & $\begin{array}{c}\text { Dose } \\
(\mathrm{g} \mathrm{i.a.} \mathrm{ha-1})\end{array}$ \\
\hline Testemunha & - & - \\
Atrazine & Atrazina Nortox 500 SC & 500 e 1000 \\
Bentazon & Basagran 600 \\
Carfentrazone & Aurora 400 EC & 720 \\
Clomazone & Gamit 360 CS & 3,75 \\
Diclosulam & Spider 840 WG & 400 \\
Diuron & Karmex & 20 \\
Ethoxysulfuron & Gladium & 800 \\
Flumioxazin & Flumyzin 500 & 18 \\
Mesotrione & Callisto & 10 \\
Oxadiazon & Ronstar 250 BR & 60 \\
Tembotrione & Soberan & 500 \\
Atrazine + flumioxazin + carfentrazone & Atrazina Nortox 500 SC + Flumyzin 500 + Aurora 400 EC & $300+5+2$ \\
\hline "Adiciona
\end{tabular}

${ }^{*}$ Adicionado adjuvantes de acordo com a recomendação do fabricante.

Os herbicidas foram aplicados aos 18 DAE da cultura, quando as plantas se encontravam no estádio de folhas unifolioladas. Para isso, foi utilizado pulverizador costal pressurizado com $\mathrm{CO}_{2}$ a $200 \mathrm{kPa}$ de pressão, com pontas jato plano tipo leque110-02, a uma altura de aproximadamente $50 \mathrm{~cm}$ em relação as plantas de crotalária e volume de calda equivalente a $300 \mathrm{~L} \mathrm{ha}^{-1}$.

As avaliações de fitotoxicidade foram realizadas aos 7, 14, 21 e 28 dias após a aplicação dos herbicidas (DAA). Utilizou-se a escala de notas proposta pela SBCPD (1995), sendo que $0 \%$ representa nenhuma injúria às plantas e $100 \%$ a morte total.

Aos 28 DAA foi realizada avaliação da matéria seca da parte aérea. Para tal avaliação as plantas foram cortadas rente ao solo, acondicionadas em sacos de papel e secas em estufa de circulação forçada de ar, a temperatura média de $72^{\circ} \mathrm{C}$, até atingir massa constante e, posteriormente, pesadas em balança analítica.

Os dados foram submetidos à análise de variância pelo teste $\mathrm{F}(\mathrm{p}<0,05)$ e as médias comparadas pelo teste de Scott-Knott $(\mathrm{p}<0,05)$. As análises estatísticas foram realizadas utilizando-se o programa SISVAR (Ferreira, 2003).

Os resultados do Experimento 1 indicam que devido a aplicação dos tratamentos herbicidas houve diferenças percentuais de fitotoxicidade na crotalária aos 7, 14, 21 e 28 dias após a aplicação (DAA) (Tabela 3). De maneira geral, independentemente da época de avalição, constatou-se baixa injúria à cultura para a aplicação de bentazon, clomazone, diclosulam, diuron e ethoxysulfuron. Tais resultados se mantiveram até os 28 DAA, na qual esses herbicidas provocaram intoxicação inferior à 23,5\%. Em trabalho de Braz et al. (2016), os autores verificaram baixos níveis de intoxicação para a aplicação de bentazon (576 e $720 \mathrm{~g} \mathrm{ha}^{-1}$ ) em pós-emergência da cultura da crotalária. Braz et al. (2015), observaram que os herbicidas bentazon e diclosulam (720 e 35,28 g ha $^{-1}$ ) aplicados em pós e pré-emergência da cultura, respectivamente, apresentaram baixos níveis de intoxicação e considerados potenciais herbicidas para esta cultura.

A crotalária apresenta grande capacidade de tolerância ao diclosulam em estudos para avaliação de espécies fitorremediadoras (Monquero et al., 2013). Braz 
et al. (2015) consideraram o herbicida diuron como não seletivo em aplicação em pós emergência da cultura da crotalária, entretanto, utilizou-se a dose de 2000 g i.a. ha ${ }^{-1}$. No presente estudo, os menores índices de intoxicação verificados podem estar relacionados a menor dose utilizada do herbicida diuron. Já para o ethoxysulfuron constatou-se baixos sintomas de fitointoxicação aplicado em pós emergência ao feijoeiro (Assis et al., 2014). Nesse mesmo estudo, os autores consideraram esse herbicida como uma ferramenta importante visando o controle de plantas voluntárias de soja.

Tabela 3. Fitointoxicação (\%) da crotalária aos 7, 14, 21 e 28 dias após a aplicação de diferentes herbicidas em pós-emergência. Experimento 1.

\begin{tabular}{lccccc}
\hline \multirow{2}{*}{ Tratamentos } & Dose & \multicolumn{4}{c}{ Fitointoxicação (\%) } \\
\cline { 3 - 6 }$\left(\mathrm{g} \mathrm{ha}^{-1} \mathrm{i} . \mathrm{a}.\right)$ & $7 \mathrm{DAA}$ & $14 \mathrm{DAA}$ & $21 \mathrm{DAA}$ & $28 \mathrm{DAA}$ \\
\hline Testemunha sem herbicida & - & $0,00 \mathrm{i}$ & $0,00 \mathrm{i}$ & $0,00 \mathrm{i}$ & $0,00 \mathrm{l}$ \\
Bentazon & 720 & $4,50 \mathrm{~h}$ & $6,50 \mathrm{~h}$ & $10,25 \mathrm{~h}$ & $12,00 \mathrm{k}$ \\
Clomazone & 400 & $14,50 \mathrm{~g}$ & $15,75 \mathrm{~g}$ & $19,75 \mathrm{~g}$ & $20,75 \mathrm{i}$ \\
Carfentrazone & 3,75 & $26,50 \mathrm{f}$ & $29,50 \mathrm{e}$ & $32,50 \mathrm{e}$ & $35,50 \mathrm{f}$ \\
Diclosulam & 20 & $5,50 \mathrm{~h}$ & $6,50 \mathrm{~h}$ & $9,50 \mathrm{~h}$ & $14,25 \mathrm{j}$ \\
Diuron & 800 & $5,50 \mathrm{~h}$ & $6,50 \mathrm{~h}$ & $9,50 \mathrm{~h}$ & $11,50 \mathrm{k}$ \\
Ethoxysulfuron & 18 & $14,50 \mathrm{~g}$ & $16,50 \mathrm{~g}$ & $18,50 \mathrm{~g}$ & $23,50 \mathrm{~h}$ \\
Flumioxazin & 10 & $15,50 \mathrm{~g}$ & $19,50 \mathrm{f}$ & $25,50 \mathrm{f}$ & $31,25 \mathrm{~g}$ \\
Atrazine & 1000 & $79,50 \mathrm{a}$ & $84,00 \mathrm{a}$ & $87,50 \mathrm{a}$ & $95,00 \mathrm{~b}$ \\
Atrazine & 500 & $79,50 \mathrm{a}$ & $85,50 \mathrm{a}$ & $86,50 \mathrm{a}$ & $99,00 \mathrm{a}$ \\
Oxadiazon & 500 & $40,50 \mathrm{c}$ & $45,50 \mathrm{c}$ & $49,50 \mathrm{c}$ & $56,50 \mathrm{~d}$ \\
Tembotrione & 40 & $29,50 \mathrm{e}$ & $34,50 \mathrm{~d}$ & $38,50 \mathrm{~d}$ & $46,00 \mathrm{e}$ \\
Mesotrione & 60 & $34,50 \mathrm{~d}$ & $44,75 \mathrm{c}$ & $49,00 \mathrm{~b}$ & $55,75 \mathrm{~d}$ \\
Atrazine + flumioxazin + carfentrazone & $300+5+2$ & $61,75 \mathrm{~b}$ & $65,50 \mathrm{~b}$ & $69,00 \mathrm{~b}$ & $74,50 \mathrm{c}$ \\
\hline Média Geral & & 29,41 & 32,89 & 36,11 & 41,10 \\
Teste F (para tratamentos) & & $1783,52^{* *}$ & $1779,21^{* *}$ & $1856,97^{* *}$ & $1363,23^{* *}$ \\
Coeficiente de variação (\%) & 4,35 & 4,13 & 3,69 & 4,14 \\
\hline
\end{tabular}

Médias seguidas de mesma letra na coluna, não diferenciam entre si pelo critério de agrupamento Scott-Knott ao nível de significância de $5 \%$ de probabilidade. ${ }^{* *}$ Significativo a $1 \%$ de probabilidade pela análise de variância.

Os maiores efeitos fitotóxicos foram observados para os herbicidas atrazine $(500 \mathrm{e}$ $\left.1000 \mathrm{~g} \mathrm{ha}{ }^{-1}\right)$, atrazine + flumioxazin + carfentrazone-ethyl, oxadiazon e mesotrione em todas as avaliações realizadas (Tabela 3 ).

A atrazine foi mais tóxica a crotalária e os sintomas semelhantes aos observados por Biffe et al. (2010), como clorose internervural e das bordas das folhas (escurecimento), que progrediram, da borda para o centro, para a necrose generalizada da folha, levando as plantas tratadas praticamente a morte. Braz et al. (2015) constataram que os herbicidas atrazine e flumioxazin aplicados em pré e pós-emergência da cultura, não foram seletivos a crotalária, por proporcionar elevados percentuais de intoxicação.
No presente estudo, a matéria seca da parte aérea (MSPA) das plantas de crotalária apresentou decréscimos superiores a $85 \%$ após a aplicação dos herbicidas atrazine (500 e 1000 $\left.\mathrm{g} \mathrm{ha}^{-1}\right)$, mesotrione, oxadiazon e atrazine + flumioxazin + carfentrazone (Figura 1). Isso indica que as reduções no acúmulo de MSPA apresentaram relação direta aos níveis de intoxicação proporcionados pelos herbicidas às plantas de crotalária.

Essa redução na MSPA e seus possíveis efeitos no desenvolvimento da planta de crotalária resulta na diminuição competitiva com outras culturas (Fialho et al., 2012), logo, o uso desses herbicidas não deve ser incentivado. Já os herbicidas bentazon, clomazone, diclosulam e ethoxysulfuron causaram reduções na MSPA próximos a $20 \%$. Os resultados 
indicam potencial de uso destes herbicidas em áreas de cultivo da crotalária, em aplicações em pós-emergência da cultura.

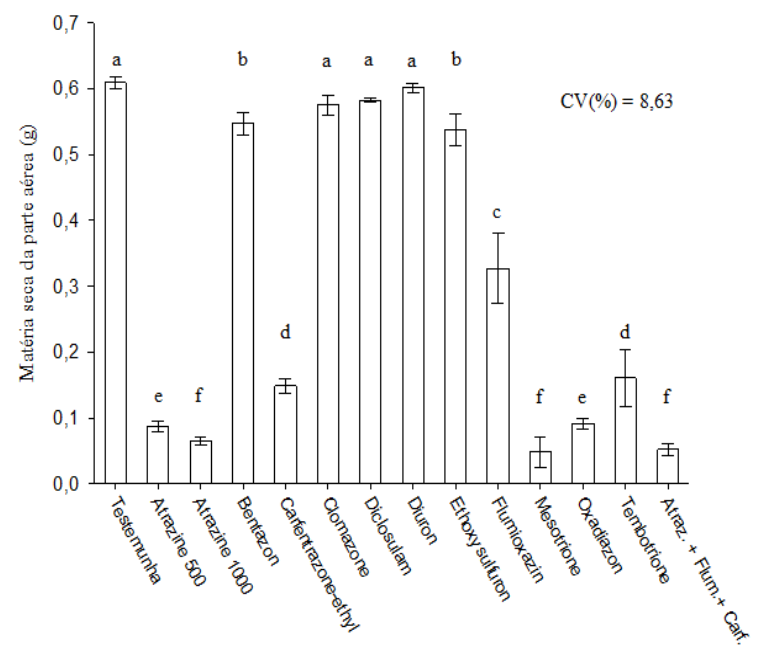

Médias seguidas de mesma letra não diferem entre pelo teste de Scott-Knott ( $p>0,05)$. Barras indicam desviopadrão da média.

Figura 1. Matéria seca da parte aérea de plantas de crotalária aos 28 DAA de diferentes herbicidas em pós-emergência da cultura (Experimento 1).

No Experimento 2, os herbicidas bentazon (400 $\left.\mathrm{g} \mathrm{ha}^{-1}\right)$, clomazone (400 $\left.\mathrm{g} \mathrm{ha}^{-1}\right)$, diclosulam (10 e $15 \mathrm{~g} \mathrm{ha}^{-1}$ ), diuron (400 $\mathrm{g} \mathrm{ha}^{-1}$ ) e ethoxysulfuron $\left(15 \mathrm{~g} \mathrm{ha}^{-1}\right)$ foram os produtos que promoveram menores índices visuais de intoxicação nas plantas de crotalária em todas as avaliações realizadas, o que corrobora com o estudo realizado no Experimento 1 (Tabela 4). A seletividade dos herbicidas bentazon e clomazone, está relacionada a mecanismos de detoxificação, por meio da ação do citocromo P450 monoxigenase (Gronwald e Connelly, 1991, Pataky, et al., 2008). Para as leguminosas como a soja e feijão, os herbicidas diclosulam e ethoxysulfuron, respectivamente, demonstraram seletividade a estas culturas (Oliveira Neto et al., 2009, Assis et al., 2014).

Ademais, o comportamento para estes herbicidas era esperado, já que foram considerados seletivos e potencias para a aplicação na cultura da crotalária (Braz et al., 2015, Braz et al., 2016). As misturas bentazon + diclosulam, diclosulam + diuron e bentazon + diuron aumentaram os sintomas de intoxicação em relação à aplicação isolada destes herbicidas. A mistura de herbicidas pode ter efeito antagônico e afetar a eficácia e a seletividade, sendo necessário conhecer a compatibilidade entre os herbicidas utilizados.

A matéria seca da parte aérea (MSPA) das plantas de crotalária apresentou redução superior a $34,5 \%$ após a aplicação dos herbicidas em mistura (Figura 2).

Tabela 4. Fitointoxicação (\%) da crotalária aos 7, 14, 21 e 28 dias após a aplicação de diferentes herbicidas em pós-emergência. Experimento 2.

\begin{tabular}{lccccc}
\hline \multirow{2}{*}{ Tratamentos } & Dose & \multicolumn{4}{c}{ Fitointoxicação (\%) } \\
\cline { 3 - 6 } & $\left(\mathrm{g} \mathrm{ha}^{-1}\right.$ i.a. $)$ & 7 DAA & $14 \mathrm{DAA}$ & $21 \mathrm{DAA}$ & $28 \mathrm{DAA}$ \\
\hline Testemunha sem herbicida & -- & $0,00 \mathrm{~g}$ & $0,00 \mathrm{~h}$ & $0,00 \mathrm{i}$ & $0,00 \mathrm{~h}$ \\
Bentazon & 400 & $5,00 \mathrm{f}$ & $12,25 \mathrm{e}$ & $14,75 \mathrm{f}$ & $19,75 \mathrm{e}$ \\
Clomazone & 400 & $9,25 \mathrm{~d}$ & $14,25 \mathrm{~d}$ & $21,75 \mathrm{~d}$ & $23,50 \mathrm{~d}$ \\
Diclosulam & 10 & $5,00 \mathrm{~g}$ & $7,50 \mathrm{~g}$ & $12,00 \mathrm{~g}$ & $14,00 \mathrm{f}$ \\
Diclosulam & 15 & $7,50 \mathrm{e}$ & $10,50 \mathrm{f}$ & $13,50 \mathrm{f}$ & $15,25 \mathrm{f}$ \\
Diuron & 400 & $5,00 \mathrm{f}$ & $7,25 \mathrm{~g}$ & $9,50 \mathrm{~h}$ & $11,75 \mathrm{~g}$ \\
Ethoxysulfuron & 15 & $9,75 \mathrm{~d}$ & $14,75 \mathrm{~d}$ & $19,50 \mathrm{e}$ & $24,00 \mathrm{~d}$ \\
Bentazon + diclosulam & $200+7,5$ & $15,00 \mathrm{c}$ & $25,00 \mathrm{c}$ & $30,00 \mathrm{c}$ & $35,00 \mathrm{c}$ \\
Diclosulam + diuron & $7,5+200$ & $30,00 \mathrm{a}$ & $35,75 \mathrm{a}$ & $47,50 \mathrm{a}$ & $62,50 \mathrm{a}$ \\
Bentazon + diuron & $200+200$ & $16,00 \mathrm{~b}$ & $27,75 \mathrm{~b}$ & $41,50 \mathrm{~b}$ & $50,50 \mathrm{~b}$ \\
\hline Média Geral & & 10,25 & 15,50 & 21,00 & 25,63 \\
Teste F (para tratamentos) & & $1007,44^{* *}$ & $463,55^{* *}$ & $586,03^{* *}$ & $942,92^{* *}$ \\
Coeficiente de variação (\%) & & 5,19 & 6,51 & 5,80 & 4,79 \\
\hline
\end{tabular}

Médias seguidas de mesma letra na coluna, não diferenciam entre si pelo critério de agrupamento Scott-Knott ao nível de significância de $5 \%$ de probabilidade. ${ }^{* *}$ Significativo a $1 \%$ de probabilidade pela análise de variância. 


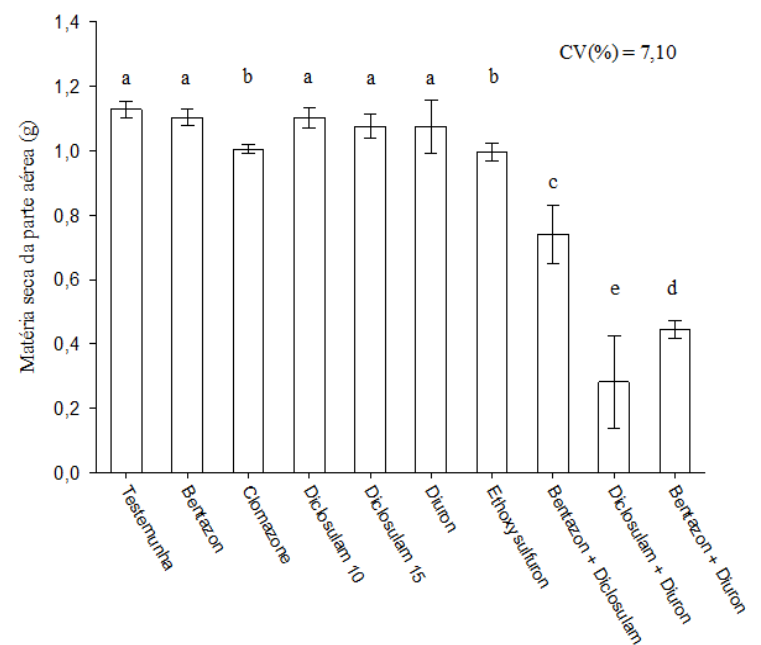

Médias seguidas de mesma letra não diferem pelo teste de Scott-Knott ( $p>0,05)$. Barras indicam desvio-padrão da média.

Figura 2. Matéria seca da parte aérea de plantas de crotalária aos 28 DAA de diferentes herbicidas em pós-emergência da cultura (Experimento 2).

O clomazone e ethoxysulfuron reduziram 11,5 e 16\%, respectivamente a MSPA, enquanto os demais herbicidas não afetaram essa variável. A maior quantidade de biomassa pode resultar em ganhos para a matéria orgânica do solo, o aumento da disponibilidade de nutrientes pela crotalária e supressão no desenvolvimento da comunidade de plantas daninhas (Timossi et al., 2011; Soratto et al., 2012).

Os herbicidas testados interferem de forma variada nas plantas de crotalária. Os herbicidas bentazon, clomazone, diclosulam e ethoxysulfuron mostram-se seletivos a cultura. A atrazine, oxadiazon, mesotrione e atrazine + flumioxazin + carfentrazone-ethyl não são seletivos à cultura.

\section{Referências}

Adler, M.J.; Chase, C.A. Comparison of the allelopathic potential of leguminous summer cover crops: cowpea, sunn hemp, and velvetbean. HortScience, v.42, n.2, p.289-293, 2007.
Assis, A.C.D.L P.; Reis, M.R.; Pessoa, G.D.O.; Silva, D.V.; Hayata, M.; Dias, R.C.; Rocha, B.H. Seletividade do ethoxysulfuron às culturas da soja e feijão. Revista Brasileira de Herbicidas, v.13, n.2, p.117-124, 2014.

Biffe, D.F.; Constantin, J.; Oliveira Jr, R.S.; Rios, F.A.; Franchini, L.H.M.; Gemelli, A. Avaliação de herbicidas para dois cultivares de mandioca. Planta Daninha, v.28, n.4, p.807816, 2010.

Braz, G.B.P.; Oliveira Jr, R.S.; Constantin, J.; Takano, H.K.; Chase, C.A.; Fornazza, F.G.F.; Raimondi, R.T. Selection of herbicides targeting the use in crop systems cultivated with showy crotalaria. Planta Daninha, v.33, n.3, p.521-534, 2015.

Braz, G.B.P.; Oliveira Jr, R.S.; Constantin, J.; Takano, H.K.; Godinho, F.B. Selectivity of herbicides applied in post-emergence of showy crotalaria. Revista Caatinga, v.29, n.4, p.918926, 2016.

Calegari, A.; Mondardo, A.; Bulisani, E.A.; Costa, M.B.B.; MIYASAKA, S.; Amado, T. J. C. Aspectos gerais da adubação verde. In: Costa, M.B B. (Coord.). Adubação verde no sul do Brasil. 2. ed. Rio de Janeiro: Assessoria e Serviços a Projetos em Agricultura Alternativa, 1993. p. 1-56.

EMPRESA BRASILEIRA DE PESQUISA AGROPECUÁRIA - EMBRAPA. Centro Nacional de Pesquisa Agropecuária de Solos (Brasília, DF). Sistema brasileiro de classificação de solos. Brasília: Embrapa Produção de Informação; Brasília, DF: Embrapa Solos, 2013. 154p.

Erasmo, E.A.L.; Azevedo, W.R.; Sarmento, R.A.; Cunha, A.M.; Garcia, S.L.R. Potencial de espécies utilizadas como adubo verde no manejo integrado de plantas daninhas. Planta Daninha, v.22, n.3, p.337-342, 2004.

Ferreira, D.F. Sisvar: a computer statistical analysis system. Ciência e Agrotecnologia, Lavras, v.35, n.6, p. 039-1042, 2011. 
Fialho, C.M.T.; Silva, A.A.; Faria, A.T.; Torres, L.G.; Rocha, P R.R.; Santos, J.B. Teor foliar de nutrientes em plantas daninhas e de café cultivadas em competição. Planta Daninha, v.30, n.1, p.65-73, 2012.

Gitti, D.C.; Arf, O.; Vilela, R.G.; Portugal, J.R.; Kaneko, F.H.; Rodrigues, R. A.F. Épocas de semeadura de crotalária em consórcio com milho. Revista Brasileira de Milho e Sorgo, v.11, n.2, p.156-168, 2012.

Gronwald, J.W.; Connelly, J.A. Effect of monooxygenase inhibitors on bentazon uptake and metabolism in maize cell suspension cultures. Pesticide Biochemistry and Physiology, v.40, n.3, p.284-294, 1991.

Meschede, D.K.; Ferreira, A.B.; Ribeiro J.R. Avaliação de diferentes coberturas na supressão de plantas daninhas no cerrado. Planta Daninha, v.25, n.3, p.465-471, 2007.

Monquero, P.A.; Amaral, L.R.; Inácio, E.M.; Brunhara, J.P.; Binha, D.P.; Silva, P.V.; Silva, A.C. Efeito de adubos verdes na supressão de espécies de plantas daninha. Planta Daninha, v.27, n.1, p.85-95, 2009.

Monquero, P.A.; Côrrea, M.C.; Barbosa, L.N.; Gutierrez, A.; Orzari, I.; Hirata, A.C.S. Seleção de espécies de adubos verdes visando à fitorremediação de diclosulam. Planta Daninha, v.31, n.1, p.127-135, 2013.

Neto, M.E.F.; Pitelli, R.A.; Basile, E.; Timossi, P.C. Seletividade de herbicidas pós-emergentes aplicados na soja geneticamente modificada. Planta Daninha, v.27, n.2, p.345-352, 2009,

Oliveira Neto, A.M.; Maciel, C.D. de G.; Guerra, N.; Lima, G.G.R.; Sola Júnior, L.C. Manejo químico de adubos verdes para sucessão da cana-de-açúcar em sistema de cultivo mínimo. Revista Brasileira de Herbicidas, v.10, n.2, p.86-94, 2011.

Pataky, J.K.; Meyer, M.D.; Bollman, J.D.; Boerboom, C.M.; Williams, M.M. Genetic basis for varied levels of injury to sweet corn hybrids from three cytochrome p450-metabolized herbicides. Journal of the American Society Horticultural Science, v.133, n.1, p.438-447, 2008.

Sagrilo, E.; Leite, L.F.C.; Galvão, S.R.S.; Lima, E.F. Manejo Agroecológico do Solo: os Benefícios da Adubação Verde. Teresina: Embrapa Meio-Norte, 2009. 24 p. (Documentos, 193).

Santos, P.A.; Silva, A.F.D.; Carvalho, M.A.C.D.; Caione, G. Adubos verdes e adubação nitrogenada em cobertura no cultivo do milho. Revista Brasileira de Milho e Sorgo, v.9, n.2, p.123-134, 2010.

Silva, T.O.; Menezes, R.S.C. Adubação orgânica da batata com esterco e, ou, Crotalaria juncea. II. Disponibilidade de N, P e K no solo ao longo do ciclo de cultivo. Revista Brasileira de Ciência do Solo, v.31, n.1, p.51-61, 2007.

Sociedade Brasileira da Ciência das Plantas Daninhas - SBCPD. Procedimentos para instalação, avaliação e análise de experimentos com herbicidas. Londrina: SBCPD, 1995. $42 \mathrm{p}$.

Soratto, R.P.; Crusciol, C.A.C.; Costa, C.H.M.D.; Ferrari Neto, J.; Castro, G.S.A. Produção, decomposição e ciclagem de nutrientes em resíduos de crotalária e milheto, cultivados solteiros e consorciados. Pesquisa Agropecuária Brasileira, v.47, n.10, p.14621470, 2012.

Timossi, P.C.; Wisintainer, C.; dos Santos, B.J.; Pereira, V.A.; Porto, V.S. Supressão de plantas daninhas e produção de sementes de crotalária, em função de métodos de semeadura. Pesquisa Agropecuária Tropical, v.41, n.4, p.525-530, 2011.

Wang, K.; Sipes, B.S.; Schimitt, D.P. Crotalaria as a cover crop for nematode management: A review. Nematropica, v.32, n.1, p.35-57, 2002. 